\title{
Reavaliação e rememoração dos conceitos da mecânica geral com análises geométricas e/ou gráficas: máquinas simples. Parte II
}

(Re-evaluation and recall of concepts of the general mechanics with geometric and/or graphics analysis: simple machines - Part II)

\author{
P.F. Barbieri ${ }^{\mathbb{M}}$ \\ Faculdade de Tecnologia de Mogi Mirim, Mogi Mirim, SP, Brazil
}

Recebido em 8/11/2010; Aceito em 29/8/2011; Publicado em 29/11/2011

\begin{abstract}
O presente trabalho analisa as relações entre forças atuantes em máquinas simples ao utilizar recursos geométricos/gráficos. São revistas algumas considerações sobre máquinas simples a fim de reforçar os conceitos físicos. Algumas dessas considerações não são abordadas na literatura básica de física geral. Para completar, foram determinadas as relações de deslocamentos para as diversas máquinas através da conservação do trabalho mecânico.
\end{abstract}

Palavras-chave: máquina simples, linha de ação de uma força, método vetorial gráfico.

This paper analyses the relationships between acting forces in simple machines through geometric/graphics features. Some considerations about simple machines are revised in order to reinforce physical concepts. These considerations are not considered in the basic literature of general physics. Thus, to complete this paper, it was determined displacement relations for various machines through to the mechanical work conservation.

Keywords: simple machines, line of action of a force, vectorial graphic methods.

\section{Introdução}

No trabalho anterior [ []] foi citado que os livros atuais de física geral possuem a tendência de atender as diversas áreas da ciência. A conseqüência disso seria que seus conteúdos ficam enxutos, omitindo assim alguns assuntos considerados fundamentais para o estudo da mecânica. Dois desses assuntos tratados de forma pouco relevante são a linha de ação de uma força e máquinas simples.

O tema máquina simples, tempos atrás tratado de forma capitular nos livros mais antigos, aparece diluído nos diversos capítulos dos livros atuais, mesmo esse sendo um dos principais assuntos motivadores para o desenvolvimento da mecânica. A omissão ou exclusão de algum tema como esse, por exemplo, pode talvez, aparecer ao custo da simplicidade ou pela mera razão de não incorrer na prolixidade, como mencionado em trabalho anterior. A lacuna que fica devido à ausência de conteúdo é refletida em cursos tecnológicos mais focados. A atual tendência de investimentos da educação brasileira em nível superior será dirigida para esses cursos tecnológicos []. Portanto, há a necessidade de trabalhar temas de ensino mais direcionados, como é da

\footnotetext{
${ }^{1}$ E-mail: paulo.barbieri@fatecmm.edu.br.

própria proposta dessa qualificação de ensino []. No entanto, a literatura formal carece de acompanhar esse processo de educação. Para os cursos tecnológicos na área da mecânica é imprescindível o estudo de máquinas simples.

Atualmente, os assuntos que englobam o tema máquina simples nos diversos capítulos que compõem os livros, não reforçam seu conceito. Como exemplo, o assunto plano inclinado e polias aparecem em con-


pecificação de máquina simples é apontada. Em outros casos tentam expor a importância com exemplos mais aplicados, $[\mathbf{G} \mathbb{Q}]$, como é o caso da máquina de Atwood. Embora seja possível compreender os motivos que conduzem à utilização de um plano inclinado, sua aplicação em sistemas diferenciados fica latente nos casos parafuso, cunha, trilhos, etc. Já no caso das polias, nenhuma explicação é dada sobre suas classificações.

Este artigo tem a proposta de rever e trabalhar em detalhes algumas das máquinas simples pouco discutidas nos livros didáticos. Considera que este assunto, além de primordial, é fundamental para o estudo da mecânica. 


\section{Trabalho de máquinas simples}

Por definição, máquina simples é aquela que não pode ser decomposta em outra. Em geral, no estudo das máquinas simples as grandezas físicas de interesse são: força potente resultante, força resistente resultante, braço mecânico de potência, braço mecânico de resistência, trabalho potente, trabalho resistente, momento torsor potente (ou torque potente), momento torsor resistente (ou torque resistente). Todavia, é observado que no estudo em particular de uma determinada máquina, apresentado por muitos livros didáticos, não se menciona a física de muitas grandezas presentes na situação em questão.

A pretensão é reforçar a idéia de que a máquina somente é o elemento de transmissão de força, independente de sua configuração. A preocupação de certa forma está concentrada nas forças, potente e resistente, que se posicionam nas "extremidades" dessas máquinas simples. Particularmente neste artigo, a força resistente sempre se configurará por uma força peso, de tal modo que o trabalho realizado pela força potente e o realizado pela força resistente serão os mesmos. Isso implicará em trabalho total nulo e permitirá estabelecer as relações de deslocamentos. Assim, a equação para o trabalho total fica

$$
W_{\text {total }}=w_{P}+w_{Q}=0
$$

onde $W_{\text {total }}$ representa o trabalho total nulo, $w_{P}$ o trabalho realizado pela força potente e $w_{Q}$ o trabalho da força resistente. Será considerado ainda, para fins de simplificação, o içar de cargas. Logo, o deslocamento de um cabo sempre será na mesma direção e sentido da força potente e, consequentemente, as cargas sempre se elevarão verticalmente em sentido oposto ao da força resistente. Considerando então que $L$ é a distância percorrida pelo cabo e $h$ a altura deslocada da carga, tem-se

$$
\begin{array}{r}
P l \cos \left(0^{\circ}\right)+Q h \cos \left(180^{\circ}\right)=0, \\
L=\frac{Q}{P} h .
\end{array}
$$

A razão $Q / P$ é denominada vantagem mecânica. Portanto, a relação entre a quantidade de cabo a ser usada pela altura a ser elevada depende da vantagem mecânica da máquina.

\section{Cunha}

A concepção de uma cunha é a mesma de um plano inclinado. Seu formato pode ser descrito como um prisma triangular como ilustrado na Fig. 1-a. Da intuição física, não é difícil ver a aplicabilidade desse instrumento e sua associação em várias ferramentas do cotidiano. Como exemplos, encontrados em muitos livros didáticos, há o calço, machado, lâminas de corte e pontas de ferramentas. A atenção para essa máquina simples está na transmissão de força, cuja compreensão reside nos conceitos de vetores.

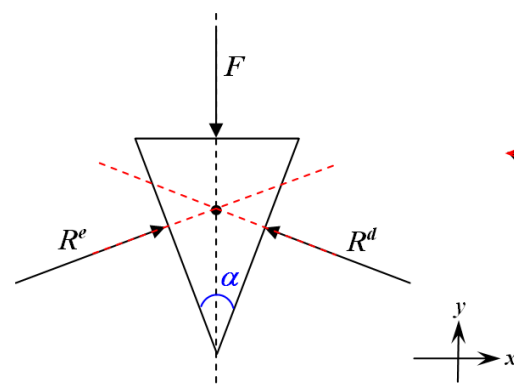

(a)

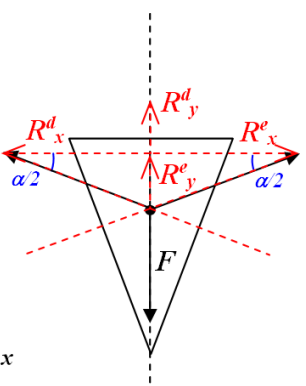

(b)
Figura 1 - (a) Cunha simétrica representada com a força aplicada $\mathbf{F}$ e as forças de resistência sobre suas laterais, lado direito $\mathbf{R}^{d}$ e lado esquerdo $\mathbf{R}^{e}$. (b) Decomposição das forças de resistência. Nota-se que na direção $\mathbf{x}$ as componentes vetoriais se cancelam, enquanto que as componentes em $\mathbf{y}$ equilibram a força aplicada.

As forças desenhadas sobre a cunha na Fig. 1-a são posicionadas de modo que suas linhas de ação interceptam-se sobre o centro de gravidade da cunha - o baricentro para o perfil mostrado. Pelo princípio de transmissibilidade e que força é um vetor deslizante [四], seus vetores então são reposicionados sobre o centro de gravidade, Fig. 1-b (força peso desprezível comparada às forças presentes, por consideração). Aplicando a segunda lei de Newton em modo vetorial inicialmente para o caso estático, é notado da figura que,

$$
\begin{gathered}
\mathbf{F}_{r}=\sum_{i=1}^{n} \mathbf{F}_{i}=0, \\
F=R_{y}^{d}+R_{y}^{e}=2 R_{y}, \\
F=2 R \operatorname{sen}\left(\frac{\alpha}{2}\right),
\end{gathered}
$$

onde $F$ é o módulo do vetor força aplicada e $R_{y}$ o módulo do vetor resistência para cada lado da cunha com intensidades iguais.

É notado da Fig. 1-b que a força de resistência é quem sofre a decomposição. Assim, racionalmente é considerado que a intensidade de $\mathbf{R}$ é maior e não a da força aplicada $\mathbf{F}$, como é conduzido em algumas literaturas que trabalham o assunto [प्व एव] - lembrando que o objetivo do plano inclinado é de reduzir o esforço realizando o mesmo trabalho. Evidentemente, não pode ser a componente maior que o próprio vetor como esses textos apresentam para a força aplicada $\mathbf{F}$.

A Eq. (6) mostra que teoricamente [ய]], para fins de transmissão de força e uma vez instalada a cunha, a força aplicada será igual o dobro da componente da resistência oferecida. Para fins mais reais e de utilidade, deve ser considerada a força de atrito. Como exemplo, a força de atrito será desfavorável na instalação de um calço. Sua orientação é contra o movimento e paralela ao eixo y (Fig. 2-a). Entretanto, com o calço já instalado e pressionado, a força de atrito será favorável a sua fixação, impedindo-o de sair, Fig. 2-b. 


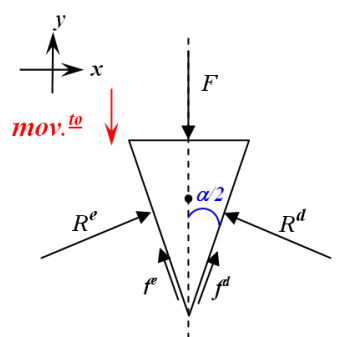

(a)

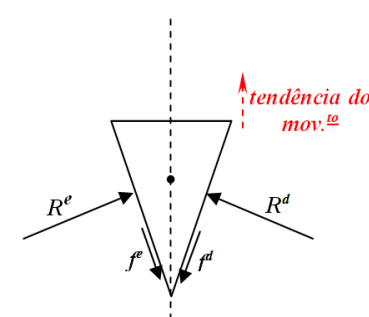

(b)

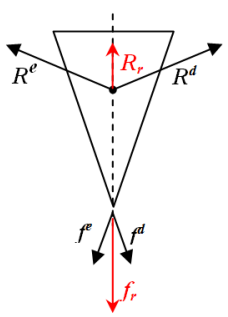

(c)

Figura 2 - (a) Instalação de um calço em forma de cunha com a representação das forças de atrito presentes nas laterais, lado direito $\mathbf{F}^{d}$ e lado esquerdo $\mathbf{F}^{e}$. (b) Calço instalado e novamente a representação das forças de atrito presentes nas laterais, mas agora com sentidos opostos a situação (a) a fim de manter a cunha presa. (c) Vetores forças resultantes para o atrito e resistência, $\mathbf{F}_{r}$ e $\mathbf{R}_{r}$, respectivamente.

Os pontos onde ocorrem as somas dos vetores na Fig. 2-c são os pontos onde as linhas de ação dos vetores em comum se interceptam. Logo, as resultantes das forças acabam por ter a mesma orientação em suas linhas de ação, eixo vertical, onde fica notável a aplicação da segunda lei de Newton.

A Eq. (7) abaixo considera a força de atrito para a instalação da cunha como calço. Adotando o sentido do movimento como positivo encontra-se

$$
\begin{gathered}
F=R_{y}^{d}+R_{y}^{e}+f_{y}^{d}+f_{y}^{d e}+m a=2\left(R_{y}+f_{y}\right)+m a \\
F=2 R\left[\operatorname{sen}\left(\frac{\alpha}{2}\right)+\mu_{c} \cos \left(\frac{\alpha}{2}\right)\right]+m a,
\end{gathered}
$$

onde $a$ é a aceleração constante sofrida pela cunha e $m$ sua massa, $f_{y}^{d}$ e $f_{y}^{e}$ são as forças de atrito para a lateral direita e esquerda, respectivamente; $\mu_{C}$ é o coeficiente de atrito cinético (instalação).

No caso da cunha já instalada é possível determinar o coeficiente de atrito mínimo exigido para a cunha não "escapar". Esse coeficiente dependerá da geometria da cunha. Segue então que, se o ângulo da ponta de ataque da cunha for $\alpha$ como na Fig. 1-a, a força de atrito pode ser escrita para a situação mínima de equilíbrio como

$$
\begin{array}{r}
f \cos \left(\frac{\alpha}{2}\right) \geq R \operatorname{sen}\left(\frac{\alpha}{2}\right), \\
\mu_{e s t} \geq \tan \left(\frac{\alpha}{2}\right),
\end{array}
$$

onde $\mu_{\text {est }}$ é o coeficiente de atrito estático. A Eq. (10) mostra explicitamente a dependência da condição mínima para o coeficiente de atrito em função da geometria da cunha. O tamanho da cunha (lateral) nada interfere, já que a força de atrito independe da área da cunha. A força peso sobre a cunha não foi considerada e para um cálculo mais exato deveria. Contudo, para levá-la em conta, dependeria da posição que a cunha se alojaria.

\section{Polias}

Quando se estuda polias pelos textos em livros didáticos não fica clara a observância das utilidades delas indepentemente de suas classificações, fixa ou móvel, seja

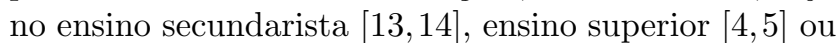

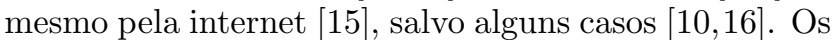
estudos dos livros textos já são conduzidos para suas combinações, ou seja, aos conjuntos de polias. Esses conjuntos também não possuem uma descrição esclarecedora. A dificuldade encontrada em qualquer nível de ensino na exploração desses assuntos já foi citada anteriormente [四].

Considere na Fig. 3 a polia "fixa que tem seu eixo preso a um suporte rígido, que lhe permite apenas o movimento de rotação, impedindo qualquer translação. As forças agem nos extremos do fio" [ए]]. Sua utilidade traz apenas comodidade, pois sua vantagem mecânica é igual a 1 , isto é, $\mathbf{P}=\mathbf{Q}$, além de permitir apenas mudar a direção do movimento. É interessante notar que as forças $\mathbf{P}$ e $\mathbf{Q}$ são realizadas no cabo, mas que podem ser associadas aos pontos de contato entre cabo e polia (nas extremidades da secção de contato). O cabo é apenas o elemento de transmissão da força.

O esforço sobre o eixo da polia deve ser igual - ou aproximadamente igual, caso o peso da própria polia e do cabo não interfira - ao dobro da tração do cabo. A polia fixa pode ser considerada como alavanca interfixa. É fácil verificar, pelos diagramas de força construídos com as linhas de ação, que a resultante atuará sobre o eixo para uma situação de equilíbrio (Fig. 3-c).

Pode ser notado da Fig. 3-c que a resultante tende na direção da força aplicada $\mathbf{P}$ em razão dessa força estar em ângulo. Para o sistema em equilíbrio a linha de ação de $\mathbf{R}$ sempre irá cruzar o eixo da polia, independente do ângulo. Assim, o ângulo criado pela força aplicada somente "desvia" a direção da força resultante, ou seja, cria um esforço maior na direção de $\mathbf{R}$. Caso $\mathbf{P}$ com $\mathbf{Q}$ tenham o mesmo ângulo de inclinação, ainda assim a linha de ação de $\mathbf{R}$ irá cruzar o eixo, mas verticalmente para baixo. 


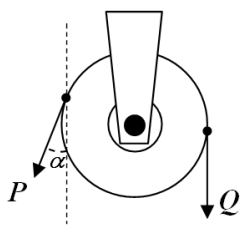

(a)

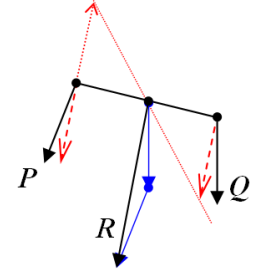

(b)

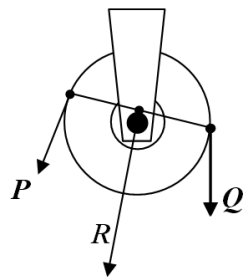

(c)
Figura 3 - (a) Polia fixa presa a um teto com as forças potente (P) e resistente $(\mathbf{Q})$ de mesma intensidade; (b) diagrama de forças com aplicação do método das componentes paralelas para determinar o ponto de ação da força resultante [U]; (c) representação das forças de tração em cada extremidade do cabo juntamente com a força resultante (R). A linha de ação de $\mathbf{R}$ cruza o eixo da polia sem efeito para o torque.

Para um caso dinâmico onde a resultante das forças não é nula, a linha de ação de $\mathbf{R}$ não deverá cruzar o eixo, porque deverá existir um torque resultante para produzir o movimento. Esse torque pode ser dado pelo produto vetorial entre o vetor $\mathbf{R}$ e a distância do ponto de atuação da própria força resultante $\mathbf{R}$ até o eixo de rotação. Isto pode ser verificado na Fig. 4.

Agora, considere a polia móvel esquematizada na Fig. 5. Ela "tem seu eixo livre, permitindo rotações e translações. Este tipo de polia é sustentado sobre o próprio fio e a força resistente (a ser superada ou equilibrada) é aplicada no eixo da polia, enquanto a força motora age no extremo livre do fio" [ए]]. As situações de análises das forças são análogas as da polia fixa, exceto que neste caso a polia móvel se compara à alavanca inter-resistente. Como destaque vale ressaltar as seguintes considerações. Primeiro, a força sobre o eixo sempre será direcionada para baixo. Segundo, de acordo com o ângulo que a força potente $\mathbf{P}$ estiver com a vertical, a polia deverá subir na orientação da força resultante $\mathbf{R}$ como mostra a Fig. 5-b e 5-c (na iminência da ação).

Para uma situação de equilíbrio com a força $\mathbf{P}$ em ângulo como na Fig. 5-a, não existiria a resultante $\mathbf{R}$, ou melhor, a resultante seria nula. Para isso, a componente vertical de $P \cos (\alpha)$ somada a $\mathbf{T}$ se anularia com Q, ou seja, aplicando a segunda lei de Newton para a situação estática,

$$
\begin{gathered}
P \cos (\alpha)+T=Q \\
2 P \cos (\alpha)=Q \\
P=\frac{Q}{2 \cos (\alpha)} .
\end{gathered}
$$

A situação da Fig. 5 trata da iminência do movimento. Porém, uma vez em movimento, a componente de $P \operatorname{sen}(\alpha)$ na horizontal seria responsável por deslocar a polia para direita e, assim, promoveria um ângulo igual para a força T. No equilíbrio, as componentes $P \operatorname{sen}(\alpha)$ e $T \operatorname{sen}(\alpha)$ se cancelariam. A Eq. (13) é uma equação geral mostrando que o ângulo limite de utilização prática do cabo é de $60^{\circ}$. A melhor situação seria para a corda paralela a resistência, $\alpha=0$, onde a força potente se reduziria a metade da resistência, isto é, $\|\mathbf{P}\|=\|\mathbf{T}\|=\|\mathbf{Q}\| / 2$.

Aplicando a conservação do trabalho ou substituindo a Eq. (13) na Eq. (3) para encontrar as relações de deslocamento, encontra-se

$$
L=2 h \cos (\alpha)
$$

onde $L$ é a extensão do cabo puxado, $h$ é o deslocamento da carga e $\alpha$ o ângulo de inclinação do cabo em relação a vertical.

\section{Conjunto de polias}

O conjunto de polias pode variar de acordo com a necessidade. No entanto, existem algumas associações bem características e que acabam recebendo a mesma denominação. São encontrados os nomes talha, moitão e cadernal, sem muita descrição de qual é um ou outro. Aqui foi denominado conforme a Fig. 6. O que deve ficar esclarecido é que, algumas associações utilizam apenas um cabo, enquanto outras utilizam mais de um, como ilustrado na Fig. 7, por exemplo.

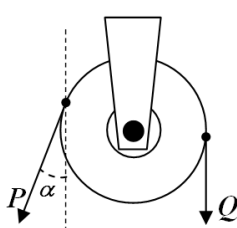

(a)



(b)

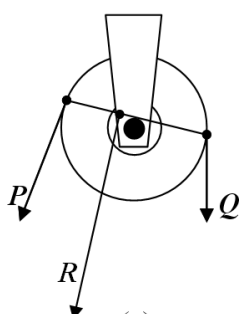

(c)
Figura 4 - (a) Polia fixa presa a um teto com as forças potente (P) e resistente ( $\mathbf{Q})$ de intensidades distintas; (b) diagrama de forças com aplicação do método das componentes paralelas para determinar o ponto de ação da força resultante [四; (c) representação das forças de tração em cada extremidade do cabo juntamente com a força resultante $(\mathbf{R})$. A linha de ação de $\mathbf{R}$ não cruza o eixo da polia produzindo torque.

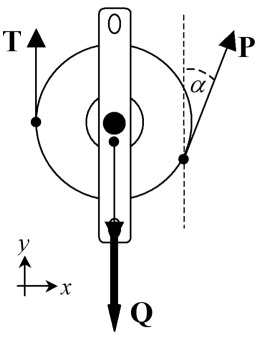

(a)

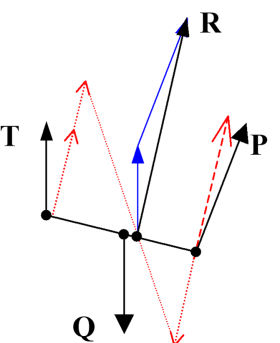

(b)

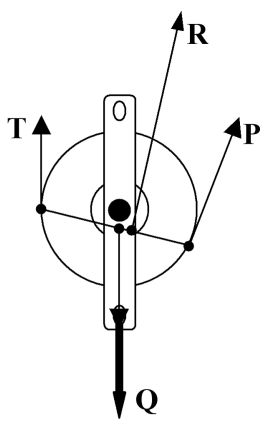

(c)
Figura 5 - (a) Polia móvel com as forças potente $(\mathbf{P})$, de tração $\mathbf{T}$ e resistente $(\mathbf{Q})$ de intensidades distintas; (b) diagrama de forças com aplicação do método das componentes paralelas para determinar o ponto de ação da força resultante [U]], neste caso, $\mathbf{R}$; (c) representação das forças de tração em cada extremidade do cabo juntamente com a força resultante $(\mathbf{R})$. A linha de ação de $\mathbf{R}$ não cruza o eixo da polia produzindo torque. 


\subsection{Associações de polias com cabo único de uma extremidade livre}

A Fig. 6 ilustra 3 tipos de associações utilizando um único cabo com o número de polias e classificações iguais.

Cada situação ilustrada na Fig. 6 mostra que, em uma situação de equilíbrio, a força resistente $\mathbf{Q}$ é suportada por quatro ramos de cabos de tensões $\mathbf{T}$ iguais (Fig. 6-d). A força potente aplicada na extremidade do cabo não deve ser levada em conta, uma vez que a polia é fixa, sendo considerado apenas um dos ramos do cabo. Como foi visto para a polia móvel em cordas paralelas $P=Q / 2$. Isso conduz à conclusão de que a força potente se divide pelo número de ramos. Seja $m$ o número de ramos de cordas, aplicando a segunda lei de Newton para o equilíbrio,

$$
m P=Q
$$

substituindo a Eq. (15) na Eq. (3) para os deslocamentos, encontra-se

$$
L=m h,
$$

onde $L$ é a extensão do cabo puxado, $h$ é o deslocamento da carga. Nos casos apresentados acima $m$ coincidirá com o número de polias.

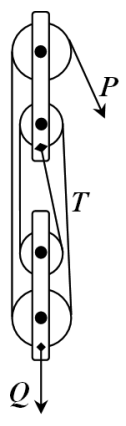

(a)

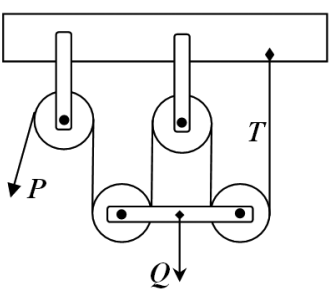

(b)

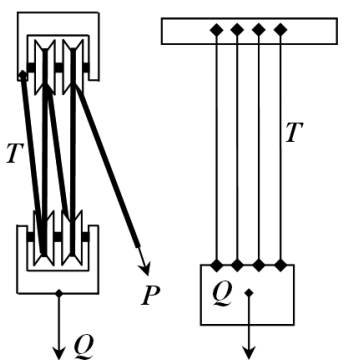

(d)
Figura 6 - Sistemas de duas polias móveis e duas fixas com cabo único. (a) Cadernal, (b) talha simples, (c) moitão e (d) diagrama esquemático mostrando situação análoga à tensão $\mathbf{T}$ do cabo para as configurações em (a), (b) e (c).

\subsection{Outras associações de polias}

Nesta seção há dois exemplos importantes. Primeiro será tratado da talha exponencial. Esta máquina configura várias polias como vários cabos. Depois, será tratado da talha diferencial. As características desta última são polias fixas e concêntricas $(A$ e $B)$ de diferentes diâmetros e seu cabo sem fim (extremidades unidas). Os esquemas dessas máquinas estão mostrados na Fig. 7.

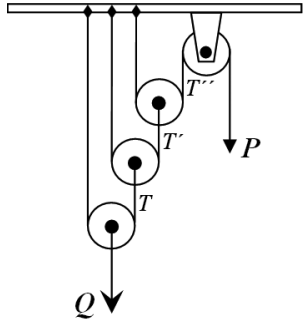

(a)

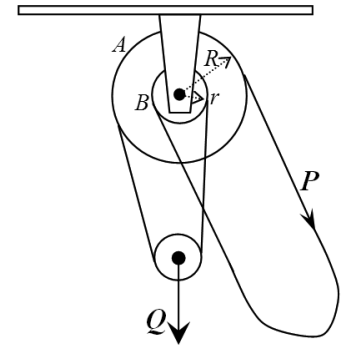

(b)
Figura 7 - (a) Esquema da talha exponencial e (b) esquema da talha diferencial.

$\mathrm{Na}$ talha exponencial, cada cabo usado tem sua tensão reduzida pela metade porque eles sempre estão trabalhando em uma polia móvel, no mínimo. Assim, para um caso geral de $n$ polias móveis e aplicando a segunda a lei de Newton para o equilíbrio, tem-se

$$
\begin{array}{r}
Q=2 T=2.2 T^{\prime}=2.2 .2 T^{\prime \prime}=2.2 .2 \ldots \\
(\text { até } n \text { vezes }) \times P=2^{n} P,
\end{array}
$$

substituindo a Eq. (17) na Eq. (3) para os deslocamentos, encontra-se

$$
L=2^{n} h
$$

onde $L$ é a extensão do cabo puxado, $h$ é o deslocamento da carga e $n$ é o número de polias móveis.

$\mathrm{Na}$ talha diferencial, as relações entre a força $\mathbf{P}$ e $\mathbf{Q}$ não são facilmente dedutíveis como anteriormente. Enquanto $\mathbf{P}$ contribuí para enrolar o cabo na polia maior, a fim de elevar a carga, contrariamente, $\mathbf{Q}$ contribui para desenrolar o cabo na polia menor (Fig. 7-b). Nessa situação não é propriamente o translado de seção do cabo unicamente que influenciará na elevação da carga. Mas, agora, a rotação das polias interfere diretamente. Então, é sensato usar a lei de equilíbrio para os torques. Como elas terão o mesmo sentido de giro, não há a necessidade de um tratamento vetorial. De modo que

$$
T_{r}=\tau_{P}^{A}-\tau_{Q}^{A}+\tau_{Q}^{B}=0
$$

onde $T_{r}$ é o torque resultante nulo, $\tau_{P}^{A}$ é o torque na polia $A$ devido a força $P, \tau_{Q}^{A}$ é o torque na polia $A$ devido a força $Q / 2$ e $\tau_{Q}^{B} A$ é o torque na polia $B$ devido a força $Q / 2$.

$$
\begin{aligned}
& P R \operatorname{sen}\left(90^{\circ}\right)-\frac{Q}{2} R \operatorname{sen}\left(90^{\circ}\right)+ \\
& \frac{Q}{2} r \operatorname{sen}\left(90^{\circ}\right)=0, \\
& P=\frac{Q(R-r)}{2 R} .
\end{aligned}
$$

Substituindo a Eq. (21) na Eq. (3) para os deslocamentos, encontra-se 


$$
L=\frac{2 R}{(R-r)} h,
$$

onde $L$ é a extensão do cabo puxado, $h$ é o deslocamento da carga, $R$ é o raio da polia fixa maior e $r$ é o raio da polia fixa menor.

\section{Sarilhos (ou molinetes)}

Sarilhos são máquinas compostas por cilindros onde se enrola um cabo. Se manual, é acoplado ao cilindro uma manivela. Primeiramente, será considerado o sarilho ordinário (único cilindro) manual e, em seguida, o sarilho diferencial manual. A Fig. 8 a seguir ilustra o esquema para estes dois sarilhos.

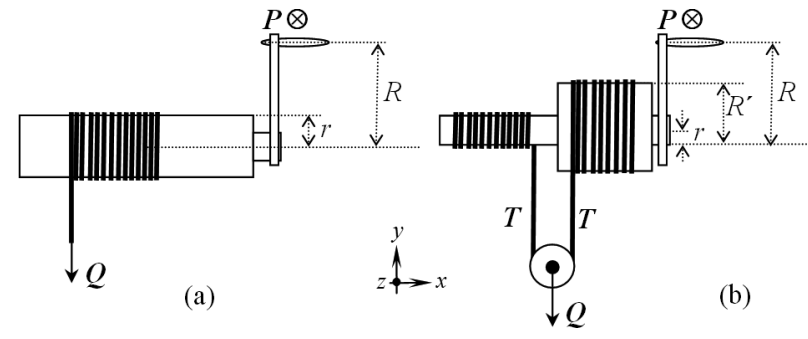

Figura 8 - a) Esquema do sarilho ordinário manual. (b) Sarilho diferencial manual: os cabos são enrolados em sentidos inversos. A força $\mathbf{P}$ está dirigida para dentro do plano da página.

Para o sarilho ordinário a relação entre força potente e resistente é estabelecida pela condição de equilíbrio nos torques. Igualmente como foi realizado para a talha diferencial os sarilhos tem a extensão dos cabos enrolados. Isso significa que as seções dos cabos estão envolvidas com a rotação do sarilho. Assim, para escrever a equação de torque resultante nulo é interessante representá-la com notação vetorial, uma vez que $\mathbf{P}$ e $\mathbf{Q}$ são ortogonais.

$$
\begin{aligned}
& \mathbf{T}_{r}=\boldsymbol{\tau}_{P}+\boldsymbol{\tau}_{Q}=0, \\
& -P R(\hat{k} \times \hat{j})-Q r(\hat{j} \times \hat{k})=0, \\
& P=Q \frac{r}{R} .
\end{aligned}
$$

Substituindo a Eq. (25) na Eq. (3) para obter a relação de deslocamento

$$
L=\frac{R}{r} h .
$$

Deve ser notado que o deslocamento $L$ da manivela do sarilho é uma seção de circunferência (Fig. 9).

Para deslocamentos infinitesimais o ângulo entre os vetores $\mathbf{L}$ e $\mathbf{P}$ tende a zero, como o trabalho é descrito por uma integral de linha, logo

$$
w_{p}=\int_{0}^{L} P d L^{\prime} \cos \left(0^{\circ}\right)=P L,
$$

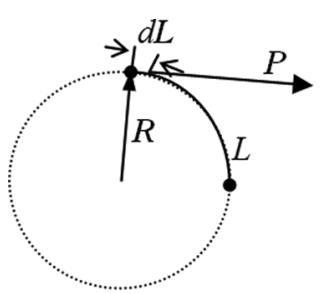

Figura 9 - Trajetória circular descrita pela manivela e o comprimento $L$ devido a um pequeno giro. Para um pequeno deslocamento $d L$ o ângulo entre os vetores $d \mathbf{L}$ e $\mathbf{P}$ é aproximadamente zero.

confirma a utilização da Eq. (3) para obtenção da Eq. (26).

Para o sarilho diferencial, do mesmo modo que a talha diferencial, deve ser aplicada a segunda lei de Newton da rotação para obter a relação entre as forças

$$
\mathbf{T}_{r}=\boldsymbol{\tau}_{P}^{R}+\boldsymbol{\tau}_{T}^{R^{\prime}}+\boldsymbol{\tau}_{T}^{r}=0,
$$

onde $\boldsymbol{\tau}_{P}^{R}$ é o torque de $\mathbf{P}$ sobre a manivela, $\boldsymbol{\tau}_{T}^{R^{\prime}}$ é o torque da tração $\mathbf{T}$ do cabo sobre o cilindro maior e $\boldsymbol{\tau}_{T}^{r}$ é o torque da tração do cabo $\mathbf{T}$ sobre cilindro menor. Como a polia que conecta os dois cilindros é uma polia móvel, extrai a relação $T=Q / 2$. Logo

$$
\begin{aligned}
& -P R \operatorname{sen}\left(90^{\circ}\right) \hat{i}+\frac{Q}{2} R^{\prime} \operatorname{sen}\left(90^{\circ}\right) \hat{i}- \\
& \frac{Q}{2} r \operatorname{sen}\left(90^{\circ}\right) \hat{i}=0, \\
& P=\frac{Q\left(R^{\prime}-r\right)}{2 R},
\end{aligned}
$$

e finalmente, substituindo a Eq. (30) na Eq. (3) para obter a relação de deslocamento

$$
L=\frac{2 R}{\left(R^{\prime}-r\right)} h
$$

\section{Discussão e conclusão}

O tema máquina simples é de grande utilidade no ensino de física e essencial para o estudo da mecânica básica. Para aqueles que seguirão no ramo da engenharia há a necessidade de uma literatura básica que verse sobre o assunto. Nos livros de física consultados de grande uso no Brasil, pouco é trabalhado do assunto, seja em ensino básico secundarista ou em ensino superior. Literaturas mais específicas que exigem que o aluno tenha cursado a disciplina de Física I ou Física Geral cobram um conhecimento prévio do assunto [ㅁ, [8]. Neste sentido, o presente artigo vem alertar sobre essa carência nos livros, como contribuir com o assunto.

Este artigo explorou as máquinas simples como um terreno imensamente interessante e rico para o fortalecimento dos conceitos físicos. Ele mostra que com o conceito de linha de ação a análise sobre uma cunha é facilitada e permite fazer outras considerações que não são exploradas, como por exemplo, a contribuição da 
força de atrito. Ainda, a literatura consultada, quando aborda o assunto da cunha mais particularmente, traz alguns conceitos confusos para sua fundamentação. Foi aqui enfatizado que a força aplicada sobre a cunha não deve ser decomposta e sim a força de resistência. Isso para não incorrer no erro da componente vetorial ser maior que o próprio vetor.

Outros conceitos que poderiam ser explorados na literatura também ficam latentes quando tratam de polias. Por exemplo, na literatura consultada apenas as vantagens mecânicas são apresentadas, ou seja, as relações entre a força potente e resistente. A pretensão com este artigo foi também mostrar outras relações interessantes que não devem passar despercebidas. Foram elas aqui denominadas de relações de deslocamentos.

Por fim, foi explorado mais enfaticamente o conceito de torque ao trabalhar os sarilhos. Neste caso, o grande interesse foi mostrar a correspondências de torque para direções de forças completamente distintas.

\section{Referências}

[1] P.F. Barbieri, Revista Brasileira de Ensino Física 33, 4304 (2011)

[2] http://www1.folha.uol.com.br/saber/799041-

institutos-de-educacao-tecnologica-terao-17-milvagas-no-sisu.shtm. Matéria do dia 15/9/2010, acesso em $28 / 10 / 2010$.

[3] http://www.jornaldaciencia.org.br/Detalhe.jsp?id=52790. Matéria do dia 4/12/2007, acesso em 28/10/2010.

[4] D. Halliday, R. Resnick e J. Walker, Fundamentos de Física (LTC, Rio de Janeiro, 2008), v. 1, $8^{\text {a }}$ ed.
[5] P.A. Tipler e G. Mosca, Física para Cientistas e Engenheiros (LTC, Rio de Janeiro, 2009), v. 1.

[6] R. Resnick, D. Halliday e K.S. Krane, Física 1 (Editora LTC, Rio de Janeiro, 2003), $5^{\mathrm{a}}$ ed.

[7] R.A. Serway, Física I para Cientistas e Engenheiros (LTC, Rio de Janeiro, 1996), $3^{\mathrm{a}}$ ed.

[8] H.D. Young e R.A. Freedman, Sears e Zemansky Física (Pearson Addison Wesley, Rio de Janeiro, 2003), $10^{\mathrm{a}}$ ed., v. 1.

[9] J. Orear, Fundamentos da Física (LTC, Rio de Janeiro, 1981), v. 1.

[10] http://www.feiradeciencias.com.br/sala06/06RE04.asp. Acesso em 30/10/2010.

[11] A. Tagliaro, Física (Editora FTD, São Paulo, 1969), v. 1 .

[12] E. Gabriades, Física (Curso apostilado, São Paulo, 1956), p. 121.

[13] J.L. Sampaio e C.S. Calçada, Física (Atual Editora, São Paulo, 2005), $2^{\text {a }}$ ed., p. 149.

[14] A.M.R. Luz e B.A. Álvares, Física (Editora Scipione, São Paulo, 2005), v. 1.

[15] http://wWw.fisica.net/mecanicaclassica/ maquinas_simples_alavancas.php. Acesso em $1 / 11 / 2010$.

[16] http://www.cienciamao.usp.br/tudo/exibir. php?mldla=pma\&cod=_pmd2005_12102. Acesso em $1 / 11 / 2010$.

[17] F.P. Beer, E.R. Johnston Jr., E.R. Eisenberg e G.H. Staab, Mecânica Vetorial para Engenheiros (McGrawHill, Rio de Janeiro, 2006), $7^{\mathrm{a}}$ ed.

[18] R.C. Hibbeler, Estática: Mecânica para Engenharia (Pearson Prentice Hall, 2005), v. 1. 